\title{
On the way to a family-centred care in neonatal units
}

\author{
Marta Canesi', \\ Davide Ausili, \\ Stefania Di Mauro ${ }^{1}$
}

${ }^{1}$ Department of Medicine and Surgery, University of Milano-Bicocca, Monza, Italy Received: 25-11-2016 Approved: 26-11-2016

Suggested citation:

Canesi M, Ausili D, Di Mauro S. On the way to a family-centred care in neonatal units.

CASUS. 2016; 1(1):11-14.
On 17 november 2016, the 6th World Prematurity Day has been celebrated. The prevalence of preterm birth is increasing worldwide. Every year, at least 1 over 10 babies are born preterm. This means 15 million preterm babies/year. Moreover, the prematurity is the second largest direct cause of deaths in children under five (1).

In the last twenty-thirty years, technological advances in neonatology have been developed and have improved the survival rate of preterm babies and critically ill ones.

Neonatal Intensive Care Unit (NICU) wards have been through several changes due to the increased number of preterm babies, who need intensive neonatology assistance and the parents' presence in the NICU to support and improve the newborn's development (1).

The prematurity can significantly affect the family wellbeing and function. The separation between the preterm newborn and the parents is a threat to the attachment and bonding process. Therefore, in the last decade, parents have been admitted together with their babies or they have been invited to spend most of the day with their babies, in the NICU. Parents in NICU reported lack of privacy, sleep disorders, dysfunctional dynamics in the parental couple, limited visitation time, restrictive touch practices, health professionals surveillance $(1,2)$.

During the NICU recovery, parents experience poor confidence as caregivers, scarce development of parenting role and lack of commitment to parental responsibilities (1). The separation of parents from their babies relates to mental health issues (e.g. depression, posttraumatic stress disorder, anxiety): they can negatively influence the baby's emotional and cognitive development. Moreover, this overall affects the social-health system: a costs increase is registered together with an higher incidence of child abuse and maltreatment following the hospital discharge. The experience of separation is even more significant and challenging for parents of very low birth weight (VLBW) newborns, who undergo intensive and prolonged treatments, leading parents to label them as "difficult" $(2,3)$. 
Physical, cognitive and psychosocial development is promoted by an early parental involvement as caregivers of their preterm babies. The Family-Centered Developmental Care (FCDC) is a further step of Family Centred Care: it aims to provide strong support to NICU families, to optimize their relationship with the newborn, in order to enhance the baby's overall development $(2,3)$. A primary goal is to minimize the long-term negative effects that a baby's illness may have on parent-baby interactions. To implement FCDC, a global culturally change is required in the entire healthcare team, together with an adjustment of NICU rules, especially referring to the historical role of professionals in the Units, aimed to increase critically ill survival and to decrease neurological impairments $(2,3)$.

Parental involvement in NICUs have been promoted through different strategies and intervention. For instance, parents have been involved in preterm babies' pain management (e.g. gentle touch, holding) and skinto-skin-care provision (Kangaroo Mother Care) (4). Several clinical outcomes have been clearly registered: improvement of infant's sleep time and temperature regulation, decreased crying and need for oxygen, decreased need of external support (e.g. ventilatory support), reduction of stressing factors that can cause an impairment in neurological development.

The baby's discharge from the NICU is experienced as one of the most stressing event and a very challenging one, because of the mixed feelings: parental anxiety combines to the going home happiness. The transition of premature infants from hospital from home life should be early planned and organized, in order to enable parents to identify their babies' needs and to be ready for home parental caregiving (5). Even if previous studies have analyzed different kind of approaches and supportive programs, further research is needed to determine the impact of some factors, such as social and cultural background, finances, education level and internal dynamics of families (1).

Teams of interdisciplinary and multidisciplinary specialists are needed to work together in order to standardize best practices in discharge, to prepare families for transition from NICU to home (3). NICU teams need expert personnel to manage the high complex needs (medical and social ones) of the family unit in order to manage different needs, reinforce the parental bonding with the baby and enhance inter-parental relationship (3). However, the relationship between health providers and parents is challenging due to a discrepancy between expectations of respective roles; previous research found out that professionals easily consider parents as "visitors" instead of primary caregivers and partners in the care process and decision making $(2,4)$. However, previous research findings showed that a positive and trustful staff-mother relationship improve the attachment to their babies, whereas a negative one may lead to disconnection and sense of isolation from them. Moreover, when mothers feel emotionally supported and have trust in their relationships with nurses, the transition from unconfident observers to competent and proactive parents is enhanced (4). A study made in UK reported that $50 \%$ 
of the participants to the study (parents of preterm infants, admitted to the NICU) did not experienced an involvement in discussions about their baby's care, even if they would have liked to; literature review findings underlined that the poor communication between parents and health professionals led to emotional crises in parents (3); 1 over 4 parent was not offered any kind of emotional support in the NICU and reported a poor understanding of their own emotional conditions, by health care providers $(3,4)$. Further research should consider (both with an observational and qualitative approach) diversities in cultural factors and resources available.

Considering a parenting support program, core crucial characteristics are (3): (1) emotional support; (2) parental education; (3) healthcare followup and (4) home visiting program.

Even if reporting about these key interventions, different support programs have been developed, with a variety of main focus, contents and implications in practice. This makes the generalizability of the findings very weak and it is hard to obtain recommendations. Research is needed to understand how to develop and implement dedicated programs, whose results and outcomes should be compared. In addiction, interventions need to be examined in depth: for example, the home visiting program should be tailored on the specific needs of the single family, according to social and health determinants that need to be clearly identified by the health care team (3).

Interdisciplinary communication, professionals collaboration, experience sharing and peer reviews are needed not only within the health care team, but also including community services providers dedicated to families with preterm babies (3). Using a "team working with teams" allows NICU professionals to align across disciplines and to take care of the entire family, while the transition from hospital to home. The health care team, thus, can help parents to identify and strengthen connections with services and resources in the community (3).

Each professional is requested to develop proper skills and knowledge, in order to implement the educational role to support parents. Optimal team collaboration requires system - level changes and support: an health system leadership supporting the interdisciplinary model of care is required to implement the FCDC in NICUs: the entire health, social and administrative staff has to be included (3). Policy makers are asked to design health and social services to take care of the preterm baby and the entire family unit, implementing coordination and integration of resources. Currently, this should be considered an imperative even relating to home palliative care, characterized by a lack of specialists and services, in the community setting. In contrast, a constant increasing number of pediatric and neonatal patients needing palliative care at home has been currently registered. 
Last, neonatal care is an expensive and limited health resource; moreover, evidence suggests that ex-preterm infants make an overuse of emergency and other health services. A structured approach to discharge planning could improve parent's self-confidence to care for their baby, facilitating earlier discharge and reducing the inappropriate use of resources (e.g. emergency services) and, then, decreasing costs. Nowadays, the issue of sustainability and equity in resource allocation is crucial and has to be faced through behavioral and cultural changes, implementing policies and professional development.

\section{REFERENCES}

1. Aagaard H, Uhrenfeldt L, Spliid M, Fegran L. Parents' experiences of transition when their infants are discharged from the Neonatal Intensive Care Unit: a systematic review protocol. JBI Database System Rev Implement Rep. 2015 ; 13(10):123-132.

2. Craig JW, Glick C, Phillips R, Hall SL, Smith J, Browne J. Reccomendations for involving the family in

\section{En camino hacia una atención centrada en la familia en las unidades neonatales} unidades neonatales developmental care of the NICU baby. J Perinatol. 2015; 35:S5-S8.

3. Purdy I.B, Craig J.W, Zeanah P. NICU discharge planning and beyond: recommendations for parent psychosocial support. J Perinatol. 2015; 35:S24-S28.

4. Finlayson K, Dixon A, Smith C, Dykes F, Flacking R. Mothers' perceptions of family centred care in neonatal intensive care units. Sex Reprod Healthc. 2014; 5:119-124.

5. Lopez G.L, Anderson K.H, Feutchinger J. Transition of Premature Infants From Hospital to Home Life. Neonatal Netw. 2012; 31(4):207-214.
El 17 de noviembre de 2016 fue el Día Mundial del Niño Prematuro. La prevalencia de nacimientos de prematuros está aumentando en todo el mundo: cada año un niño de cada diez nace antes de la semana número 37 de gestación. Se hace referencia a 15 millones de niños prematuros al año. La prematuridad es también la segunda causa principal de muerte en menores de cinco años (1).

En los últimos 20-30 años, los avances tecnológicos en neonatología han asegurado un aumento en la tasa de supervivencia de los bebés prematuros y de aquellos gravemente comprometidos. Además del aumento de lactantes severamente prematuros y que requieren significativo apoyo neonatológico, la Unidad de Cuidados Intensivos Neonatales (UCIN) ha sufrido otro cambio importante relacionado con la presencia de los padres en las unidades operativas (UO), lo cual tiene repercusiones en el desarrollo del niño (1).

La prematuridad es, de hecho, un factor que afecta en gran medida el funcionamiento y el bienestar de la familia. La separación entre padres e hijos, inducida por la hospitalización, interfiere con el proceso de apego. Como resultado la tendencia ha sido, en la última década, a hospitalizar siempre que sea posible, los padres con el niño o de invitar a los padres a pasar la mayor parte del día en la UCIN. La estancia en la misma, sin embargo, también parece tener efectos negativos para los padres, como la falta de privacidad y la sensación de estar bajo observación, la reducción de la cantidad y calidad del sueño, así como la alteración significativa de la dinámica interna de la pareja $(1,2)$. 
Durante la hospitalización del recién nacido en la UCIN, los padres tienden a perder la confianza en su papel y tienen dificultades para adquirir sus responsabilidades (1). Además, la separación de sus hijos, les puede llevar trastornos psiquiátricos (depresión, trastorno de estrés postraumático, ansiedad) que tienen repercusiones en el desarrollo psico-emocional del niño y en todo el sistema sociosanitario: además del aumento de los costos, se incrementa también el riesgo de abuso y de maltrato infantil en el hogar. La experiencia de la separación es particularmente dramática con los recién nacidos con muy bajo peso al nacer (very low birth weight VLBW), sometidos a estancias hospitalarias más prolongadas y tratamientos más intensivos, lo que lleva a los padres a etiquetar a sus hijos como "difíciles" $(2,3)$.

Recientes estudios demuestran como la participación precoz de la familia en la atención del recién nacido determina efectos positivos a largo plazo sobre el desarrollo del prematuro, desde un punto de vista físico, cognitivo y emocional. El modelo de la Family-Centered Developmental Care (FCDC) se presenta como un desarrollo del Family Centred Care (cuidado centrado en la familia) y proporciona un fuerte apoyo a las familias en la UCIN, para optimizar su relación con el bebé, favoreciendo su desarrollo general $(2,3)$. Otro objetivo es reducir al mínimo los efectos negativos que la enfermedad del niño puede tener sobre las interacciones entre padres e hijo. La implementación de este modelo requiere un cambio cultural de todo el equipo de salud, una adaptación de la organización interna de la UCIN y de su reglamento, así como una evolución del papel que históricamente han tenido los profesionales dentro de la misma (2), o sea dirigido a mejorar la supervivencia de los bebés con alto riesgo y a reducir las secuelas neurológicas (3).

Se han desarrollado estrategias y acciones destinadas a promover la participación de los padres en la UCIN, con manifiestos resultados en diferentes áreas. Por ejemplo, los padres están involucrados en el manejo del dolor del bebé prematuro (ej. gentle touch, la contención, la succión) y en la ejecución del método madre canguro (MMC)(4). Los beneficios también son significativos desde el punto de vista clínico: la mejora general de los parámetros vitales, con la posibilidad de reducir los medios de soporte externo (por ejemplo, el apoyo ventilatorio) y la reducción de los estímulos estresantes para el niño, que pueden conducir al deterioro del desarrollo del cerebro.

Por otro lado, el alta se reporta en literatura como el momento que los padres viven como más estresante, debido a que experimentan una mezcla de sentimientos contrastantes (ansiedad parental y felicidad por el éxito alcanzado con el nacimiento de su hijo). Nace la pregunta si la transición a la casa deba ser administrada en modo anticipado y de una manera estructurada, según sea necesario. Los padres de bebés prematuros, de hecho, deben ser capaces de entender las necesidades de sus hijos y estar preparados para el home parental caregiving (5). Algunos autores han propuesto protocolos a tal fin, sin embargo deben llevarse a cabo investigaciones adicionales para evaluar diversos contextos geográficos, socio-culturales, económicos y diferentes estructuras familiares (1). 
Es indispensable formar a un grupo de especialistas a nivel inter, multi e intra-disciplinar que colaboren en la estandarización de buenas prácticas en la transición de la familia del hospital al hogar (3). Dentro del equipo, es necesaria la presencia de personal experto dedicado a la gestión de las numerosas necesidades sociales y de salud de la unidad familiar y de involucrar en este proceso a la familia, a fin de estimular el vínculo interno a la pareja y de los padres con su hijo (3). Algunos estudios, de hecho, señalan que el personal médico tiene dificultades, a menudo, en el tener en cuenta los padres como colaboradores (partners) en el curso del tratamiento, considerándolos como participantes o visitantes $(2,4)$. Por otra parte, estudios han demostrado que una relación de confianza entre el personal y las madres promueve la unión con el bebé, mientras que una relación negativa conduce a la separación y al aislamiento. Cuando las madres se sienten apoyadas desde el punto de vista emocional y tienen confianza en el personal de enfermería, se favorece la transición de la sensación de ser espectadores impotentes a padres competentes y seguros (4). Un informe británico encontró que, a pesar de su deseo, la mitad de los participantes (padres de bebés prematuros ingresados en UCIN) no son involucrados en la discusión del proceso de atención de su hijo. Una revisión de la literatura (3) evidenció que la brecha de comunicación entre los padres y el equipo de cuidado médico determina crisis emocionales significativas en los primeros. Uno de cada cuatro padres informó que no había recibido ningún apoyo psico-emocional en la UCIN y de no ser comprendido por el personal médico y de enfermería $(3,4)$. Adicionales estudios observacionales o de análisis cualitativo serían útiles para evaluar diferentes contextos desde un punto de vista cultural y de los recursos disponibles.

Los hitos de un programa de apoyo de los padres son (3): (1) el apoyo emocional; (2) la educación de los padres; (3) el seguimiento de los cuidados y (4) el programa de visitas al hogar. A pesar de estas características comunes de estructura, los programas que se encuentran en la literatura difieren en objetivos, en contenidos y en la aplicación, lo que luego hace que sea difícil obtener recomendaciones generalizables. Las investigaciones futuras deberían centrarse en la ejecución de los programas y su evaluación comparativa. Deberían ser sometidas a un análisis más detallado las características específicas de las distintas intervenciones: por ejemplo, en comparación con el programa de visitas domiciliarias (home visiting). La identificación precoz de las familias en situación de riesgo permite programar la intervención de específico personal de salud, de acuerdo a las necesidades identificadas (3).

Es esencial que todo el personal busque ocasiones para el intercambio y la comunicación con otros profesionales, proveedores de servicios y programas dedicados a estas familias, sobretodo en el territorio (3). El uso de un enfoque de "equipo que trabaja con equipos" permite a los profesionales alinearse y coordinar el cuidado de la familia, a través de las diversas disciplinas. De esta manera, la familia es capaz de crear vínculos entre el contexto hospitalario y el territorial (3). 
Si bien a cada profesional del sector se le pide conocimiento profundo de sus responsabilidades y desarrollo de habilidades relacionadas con el papel educativo y de apoyo a los padres. El apoyo organizativo, sin embargo, es sin duda esencial: un liderazgo en el sistema de salud que apoye un modelo de atención multidisciplinar permite implementar la FCDC en la UCIN, con la participación del personal socio-sanitario y administrativo (3). A los decisores políticos se les requiere para organizar los sistemas y servicios socio-sanitarios en el cuidado total del bebé prematuro y de su familia. Esto es necesario y crucial también en lo que se refiere a la cuestión de los cuidados paliativos en la casa, que difícilmente encuentran servicios y personal especializado en el territorio, en comparación con una demanda cada vez mayor, incluso para la edad neonatal.

Por último, estudios publicados recientemente han analizado el impacto económico de un sistema de atención neonatal centrado en la familia. Teniendo en cuenta el tema de la sostenibilidad y de la gestión equitativa de los recursos, cada vez más relevante en la actualidad, se trata de evaluaciones de considerable sustancia e impacto, frente a las cuales no se puede permanecer indiferentes.

\section{REFERENCIAS BIBLIOGRÁFICAS}

1. Aagaard H, Uhrenfeldt L, Spliid M, Fegran L. Parents' experiences of transition when their infants are discharged from the Neonatal Intensive Care Unit: a systematic review protocol. JBI Database System Rev Implement Rep. 2015 ; 13(10):123-132.

2. Craig JW, Glick C, Phillips R, Hall SL, Smith J, Browne J. Reccomendations for involving the family in developmental care of the NICU baby. J Perinatol. 2015; 35:S5-S8.

3. Purdy I.B, Craig J.W, Zeanah P. NICU discharge planning and beyond: recommendations for parent psychosocial support. J Perinatol. 2015;35:S24-S28.

4. Finlayson K, Dixon A, Smith C, Dykes F, Flacking R. Mothers' perceptions of family centred care in neonatal intensive care units. Sex Reprod Healthc. 2014; 5:119-124.

5. Lopez G.L, Anderson K.H, Feutchinger J. Transition of Premature Infants From Hospital to Home Life. Neonatal Netw. 2012; 31(4):207-214. 